\title{
Compact Jets in 100 AGNs with the Strongest Broad-Band Variability of 1-22 GHz Spectra in 1997-2001
}

\author{
Yu. A. Kovalev' ${ }^{1}$, Y. Y. Kovalev' ${ }^{1}$, N. A. Nizhelsky ${ }^{2}$, A. V. Bogdantsov ${ }^{2}$ \\ ${ }^{1}$ Astro Space Center of the Lebedev Physical Institute RAS, \\ Profsoyuznaya 84/32, 117997 Moscow, Russia \\ ${ }^{2}$ Special Astrophysical Observatory RAS, Nizhnij Arkhyz, 369167 Russia
}

\begin{abstract}
Results of monitoring observations at the radio telescope RATAN-600 and a model interpretation of instantaneous 1-22 GHz spectra at six frequencies for 100 selected AGNs are presented. The index of variability at these frequencies is shown for 550 sources monitored in 1997-2001 at 11 epochs. The spectra of the selected sources exhibit flux density variations of about $50 \%$ and up. The type of spectral evolution is similar for all the selected objects, favoring the same basic physical model. Model analysis shows that the nature of the radio sources and the observed variability behavior of the spectra can be explained by a model with a relativistic jet of parsec scale in a longitudinal magnetic field.
\end{abstract}

\section{Observations and Results of Analysis}

Since 1997, we have monitored instantaneous 6-frequency 1-22 GHz spectra of 550 compact extragalactic radio sources with milliarcsecond components. Monitoring sets take place several times per year at the RATAN -600 ring radio telescope. The sources were selected from the Preston et al. (1985) VLBI survey. Every 1-22 GHz spectrum was measured at six wavelengths of 1.4, 2.7, $3.9,7.7,13$, and $31 \mathrm{~cm}$ over a period of a few minutes (see details in Kovalev et al. 1999).

From the distributions of 550 sources over the $V_{\nu}=\frac{S_{\nu, \max }-S_{\nu, \min }}{S_{\nu, \max }+S_{\nu, \min }}$ variability indexes at $22-2.3 \mathrm{GHz}$ over the four-year period (1997, March, - 2001, May; 11 epochs; Fig. 1) it follows that: (i) the distributions have one peak at indexes of about 0.17 at $22 \mathrm{GHz}$ and $0.10-0.05$ at $11-2.3 \mathrm{GHz}$; (ii) the mean index of variability for the full sample decreases as $0.24,0.19,0.19,0.15,0.15$ with decreasing frequency; (iii) more than $20 \%$ of the sources exhibited very strong variability at the higher frequencies in this period: with 1.5-15 times greater changes in the flux $\left(V_{\nu}\right.$ is more than 0.25$)$.

The 100 sources with the strongest variability $\left(V_{\nu}>0.25\right.$, see Fig. 1$)$ ever observed have been selected from the 550 objects monitored. The behavior of the spectra is similar for the 100 AGNs selected: a wave of a perturbation moves along the spectrum from higher to lower frequencies. As a result, a spectrum can be observed as a variable one in the entire 1-22 $\mathrm{GHz}$ band or only in the higher frequency part of the band (see examples in Fig. 1). 

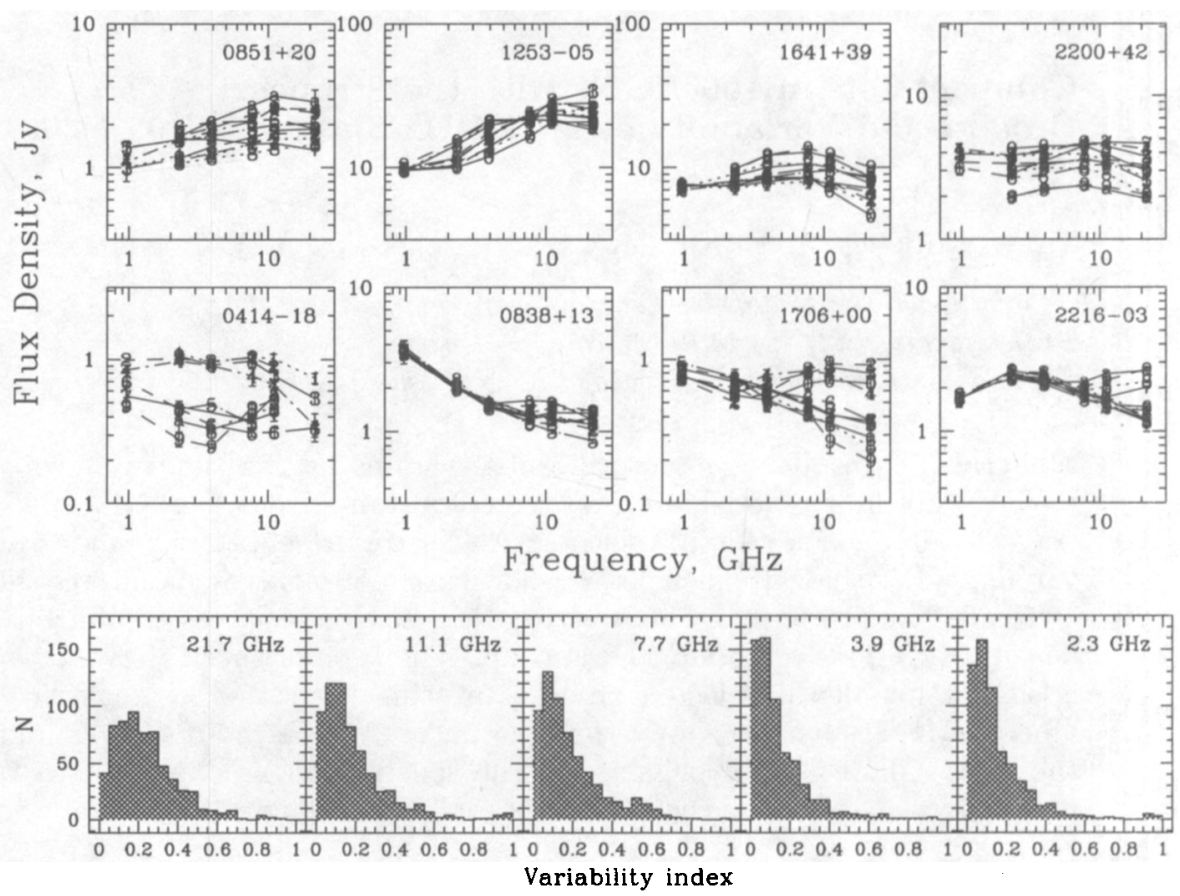

Figure 1. On the top: typical examples of multi-epoch instantaneous spectra for 8 of 100 AGNs with the strongest variability in 1997-2001. Epochs: 1997, March, June, September, December; 1998, March; 1999, April, September; 2000, April, August; 2001, March, May (11 epochs, labeled 0-9, a). On the bottom: histogram of indexes of the long-term variability at $22-2.3 \mathrm{GHz}$ for 550 VLBI compact extragalactic radio sources monitored during the same 11 epochs.

Such behavior of the variable spectra and the variability index-frequency statistical dependence can be explained by a variability of the flow of the emitting particles at the beginning of a jet in a relativistic jet model with a quasi-radial magnetic field (for details of the model interpretation, see Kovalev et al. 2000).

Acknowledgments. This work has been partly supported by the Russian State Program "Astronomy" (project 1.2.5.1), the NASA JURRISS Program (project W-19611) and the Russian Foundation for Basic Research (projects 99-02-17799, 01-02-16812, 01-02-06084).

\section{References}

Kovalev, Y.Y., Nizhelsky, N.A., Kovalev, Yu.A., et al. 1999, A\&AS, 139, 545

Kovalev, Yu.A., Kovalev, Y.Y., and Nizhelsky, N.A. 2000, PASJ, 52, 1027

Preston, R.A., Morabito, D.D., Williams, J.G., et al. 1985, AJ, 90, 1599 